\title{
FREE RADICALS IN K AND Rb ADMIXTURED FULLERENE $\mathrm{C}_{60}$
}

\author{
J. STANKoWSKI ${ }^{a}$, W. KemPińsKi ${ }^{a}$, P. BYSZEWSKI ${ }^{b}$ AND Z. TRYBUla ${ }^{a}$ \\ anstitute of Molecular Physics, Polish Academy of Sciences \\ Smoluchowskiego 17, 60-179 Poznań, Poland \\ ${ }^{b}$ Institute of Physics, Polish Academy of Sciences \\ Al. Lotników 32/46, 02-668 Warszawa, Poland
}

(Received July 12, 1993; in final form October 11, 1993)

\begin{abstract}
Molecules of $\mathrm{C}_{60}$ form Van der Waals type crystals. The observations reported here concern EPR investigations of such $\mathrm{C}_{60}$ samples with only a slight amount of $\mathrm{Rb}$ or $\mathrm{K}$. Detected defects are $\left(\dot{\mathrm{C}}_{60}\right)^{+}$or $\left(\dot{\mathrm{C}}_{60}\right)^{y-}$ ion-radicals. A hole (h) or trapped electron ( $t$ ) are localized on one fullerene ball. In the case of potassium fullerites $\mathrm{C}_{60}: K$ the line shape of EPR signal was of the Dysonian form which is characteristic of conducting electrons in metal. The EPR lines of holes $\left(\dot{\mathrm{C}}_{60}\right)^{+}$are characterized by $g_{\mathrm{h}} \geq g_{0}$ whereas for electrons $g_{t}$ is below the value $g_{0}=2.0023$ characteristic of a free electron $\left(g_{\mathrm{t}} \leq g_{0}\right)$. The EPR linewidth $2 \Delta B_{\mathbf{1 s}_{\mathrm{s}}}^{\mathrm{h}}$ of the $\left(\dot{\mathrm{C}}_{60}\right)^{+}$weakly increased with decreasing temperature whereas the EPR linewidth attributed to the electron $2 \Delta B_{1 s}^{\mathrm{t}}$ significantly decreases with decreasing temperature. The $\mathrm{C}_{60}: \mathrm{K}$ sample reached superconducting phase below $T_{\mathrm{c}}=11 \mathrm{~K}$ which is significantly less than $T_{c}=16.5 \mathrm{~K}$ observed for $\mathrm{K}_{x} \mathrm{C}_{60}$ where $2 \leq x \leq 4$.
\end{abstract}

PACS numbers: 76.30.Rn, 74.70.Wz

\section{Introduction}

EPR investigation of fullerene $\mathrm{C}_{60}$ samples proved the presence of two kinds of paramagnetic centers $[1,2]$ which differ essentially in their temperature behavior:

1. The holes $(\mathrm{h})$ induced by ionization of fullerene molecule $\left(\dot{\mathrm{C}}_{60}\right)^{1+}$ or trapped $(t)$ electrons on $\mathrm{C}_{60}$ ball due to anion radical $\left(\dot{\mathrm{C}}_{60}\right)^{1-}$. These centers present at small concentrations in pure fullerene samples [1] and their EPR spectra weakly depend on temperature. For both centers the EPR lines are narrow till the lowest temperatures $(4.2 \mathrm{~K})$ but $g$-factors are different which allows to distinguish them clearly: the former are of $g_{\mathrm{h}} \geq g_{0}=2.0023$ while those of the latter are characterized by $g_{\mathrm{t}} \leq g_{0}$, in the vicinity of $g=2.0000$. 
2. Anion radicals $\left(\dot{\mathrm{C}}_{60}\right)^{y-}$ with valence $y$ occur as complexes $\mathrm{Me}^{1+}\left(\dot{\mathrm{C}}_{60}\right)^{y-}$ whose electron structure changes with temperature. Their EPR linewidth and $g$-factor value strongly depend on temperature. This dependence is a consequence of charge instability of the $\mathrm{Me}^{1+}\left(\dot{\mathrm{C}}_{60}\right)^{y-}$. The complexes change their valence which diminishes with decreasing temperature.

So far, literature reports have concerned large concentrations of alkali metal fullerites $\mathrm{Me}_{x} \mathrm{C}_{60}$ where $x=2,3,4,5$ [1-5]. This paper presents the results of EPR investigation of $\mathrm{C}_{60}$ spectra and conclusions connected with the process of charge transfer from the alkali metal to the fullerene ball in samples of small concentration of admixtured metal $\mathrm{Me}=\mathrm{K}$ or $\mathrm{Rb}$.

\section{Experimental}

Fullerene $\mathrm{C}_{60}$ samples admixtured with a slight amount of $\mathrm{Rb}$ or $\mathrm{K}$ were subjected to EPR investigation. The samples were prepared by the method similar to that described in [1]. The soot to synthesize fullerene were generated by the contact between graphite electrodes in the reaction chamber filled with helium up to $0.2 \mathrm{~atm}$. Toluene was used as a solvent and $\mathrm{C}_{60}$ were extracted from solution with the liquid chromatography method. The suitable mass of alkali metal was added to $\mathrm{C}_{60}$ placed in a quartz tube with glass capillaries (of diameter about

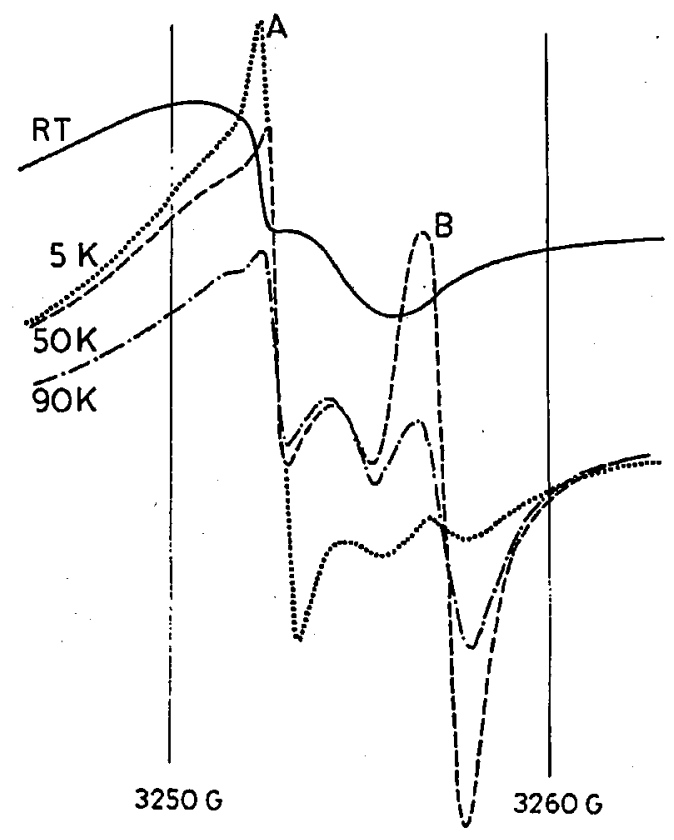

Fig. 1. EPR spectrum recorded for $\mathrm{C}_{60}: \mathrm{Rb}$. The narrow $(A)$ and wide lines are observed at room temperature. Line $B$ appears for $T<120 \mathrm{~K}$ and has the highest amplitude at $T \approx 40 \mathrm{~K}$. 
$0.5 \mathrm{~mm}$ ). The quartz tubes were vacuumed and sealed. Diffusion of the dopants was performed during two weeks in a temperature of $250^{\circ} \mathrm{C}$. Concentrations $x$ of our $\mathrm{Me}_{x} \mathrm{C}_{60}$ samples were of no more than 0.5 - part of alkali metal could be stopped on the bound of grains, therefore we will write our compounds $\mathrm{C}_{60}: \mathrm{Me}$ where $\mathrm{Me}=\mathrm{K}$ or $\mathrm{Rb}$. The spectra of samples admixtured with different alkali metals ( $\mathrm{Rb}$ or $\mathrm{K}$ ) differed drastically.

The EPR spectrum of $\mathrm{C}_{60}: \mathrm{Rb}$ taken at room temperature was evidently composed of two components: a narrow (N) line $A$ (Fig. 1) of $g^{\mathrm{N}}=2.0024$ and the width $2 \Delta B_{1 \mathrm{~s}}^{\mathrm{N}}=0.5 \mathrm{Gs}$ attributed to $\left(\dot{\mathrm{C}}_{60}\right)^{+}$ion-radical and a wide (W) line $B$ of $g^{\mathrm{W}}=2.0016$ and the width $2 \Delta B_{1 \mathrm{~s}}^{\mathrm{W}}=7 \mathrm{Gs}$ attributed to $\left(\dot{\mathrm{C}}_{60}\right)^{y-}$ ion-radical in the $\mathrm{Rb}-\mathrm{C}_{60}$ complex [1]. When temperature was decreased below $T=120 \mathrm{~K}$, line $B$ characterized by $g=1.9998$ appeared. At $50 \mathrm{~K}$ its amplitude of the first derivatives of the absorption intensity $I_{B}^{\prime}$ was twice higher than that of the initial line $A, I_{A}^{\prime}$, $\left(I_{B}^{\prime} / I_{A}^{\prime}=2\right)$, and at $40 \mathrm{~K}, I_{B}^{\prime}$ reached a maximum. With further decreasing of temperature line $B$ of $I_{B}^{\prime}$ disappeared while the intensity of the initial line $A, I_{A}^{\prime}$ was increasing according to the Curie law (Fig. 2).

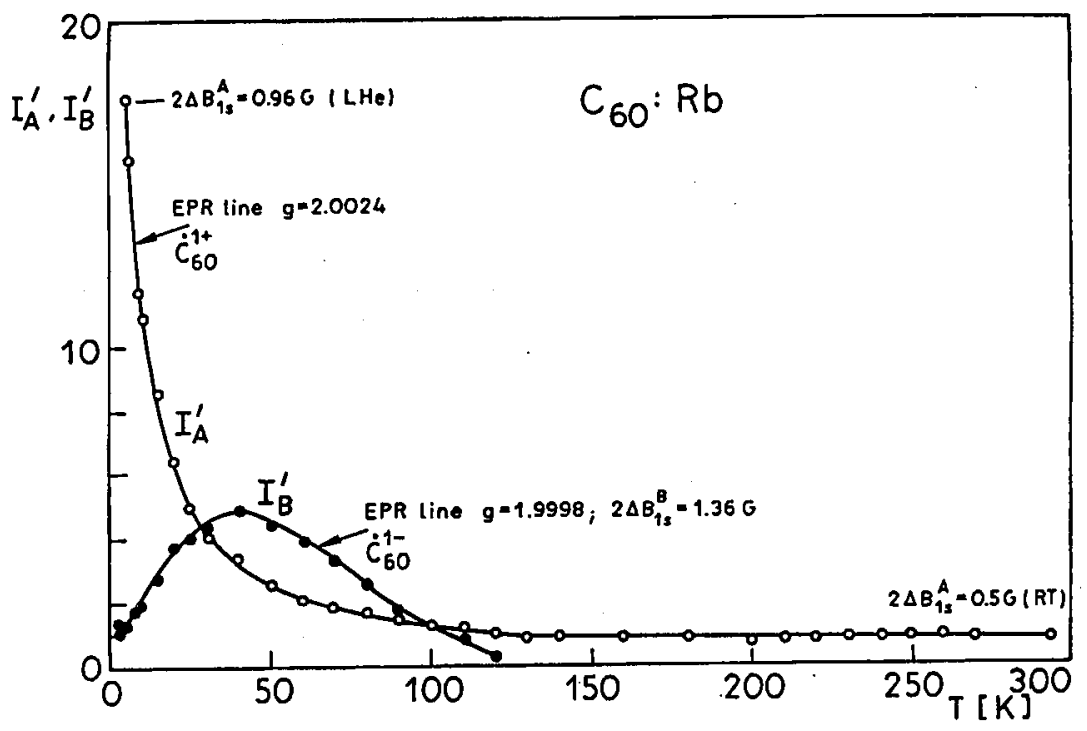

Fig. 2. Temperature dependencies of the amplitude of the first derivatives of the absorption intensity $I_{A}^{\prime}$ and $I_{B}^{\prime}$ in the EPR spectrum of $\mathrm{C}_{60}: \mathrm{Rb}$ fullerene .

The spectrum of the sample admixtured with potassium was also composed of two components: the narrow (N) line (marked as $H$ too in Figs. 3 and 4) characterized by $g^{\mathrm{N}}=2.0028$ and the width $2 \Delta B_{1 \mathrm{~s}}^{\mathrm{N}}=1.36$ Gs and the wide one (W) characterized by $g^{\mathrm{W}}=2.0020$ and $2 \Delta B_{1 \mathrm{~s}}^{\mathrm{W}}=20$ Gs. The $g$-factor of the narrow line did not change with temperature $g^{N}=$ const but the amplitude of this line increased with decreasing temperature. Below $80 \mathrm{~K}$ we could not observe this line because of strong increase in the wide line (Fig. 3 and Fig. 4). On the 
other hand, the width of wide line was reduced to $2 \Delta B_{1 \mathrm{~s}}^{\mathrm{W}}=2$ Gs at $25 \mathrm{~K}$. At this temperature $g^{\mathrm{W}}=2.0010$ and the sample became evidently metallic as its EPR line took typical Dyson's shape. Temperature dependence of the amplitudes of the components of lines $\alpha$ and $\beta$ are shown in Fig. 4. Starting from $40 \mathrm{~K}$ the line becomes asymmetric and this asymmetry increases with temperature decreasing to that of liquid helium.

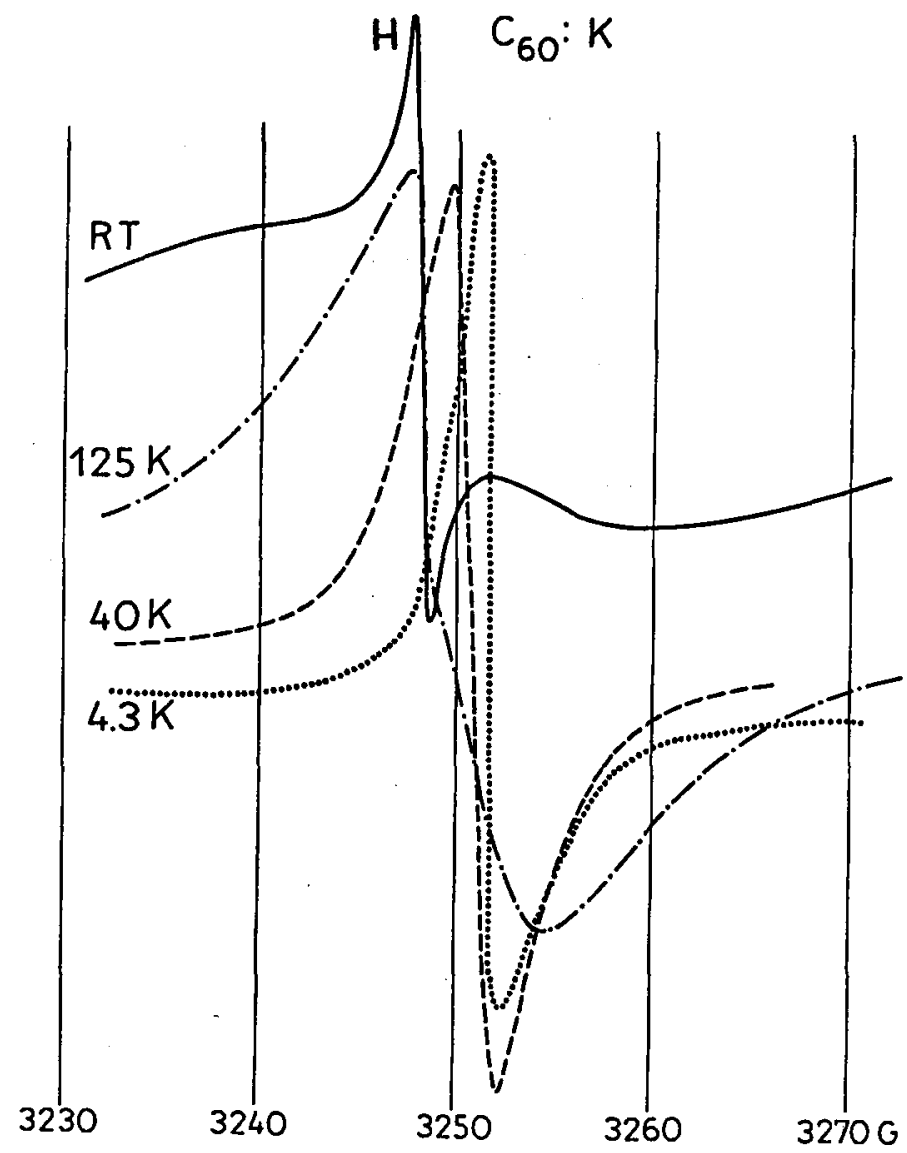

Fig. 3. Two-component EPR spectrum recorded for $\mathrm{C}_{60}: \mathrm{K}$. The narrow (line $H$ ) and wide lines are observed at room temperature. With decreasing temperature line $H$ disappears (see Fig. 4) while the wide line becomes the Dyson type line of growing $\alpha / \beta$ asymmetry.

The sample of $\mathrm{C}_{60}: \mathrm{K}$ proved superconducting below $T_{\mathrm{c}}=11 \mathrm{~K}$ whereas the critical temperature of the $\mathrm{K}_{x} \mathrm{C}_{60}$ samples for $2 \leq x \leq 4$ was $T_{\mathrm{c}}=16.5 \mathrm{~K}$ [1]. Figure 5 presents a temperature dependence of magnetic modulated microwave absorption (MMMA) signal for $\mathrm{C}_{60}: \mathrm{K}$ samples. A non-monotonous increase in $\mathrm{d} P / \mathrm{d} B$ with decreasing temperature of the sample is clearly evident.

For $\mathrm{C}_{60}: \mathrm{Rb}$ we did not observe superconducting phase. 


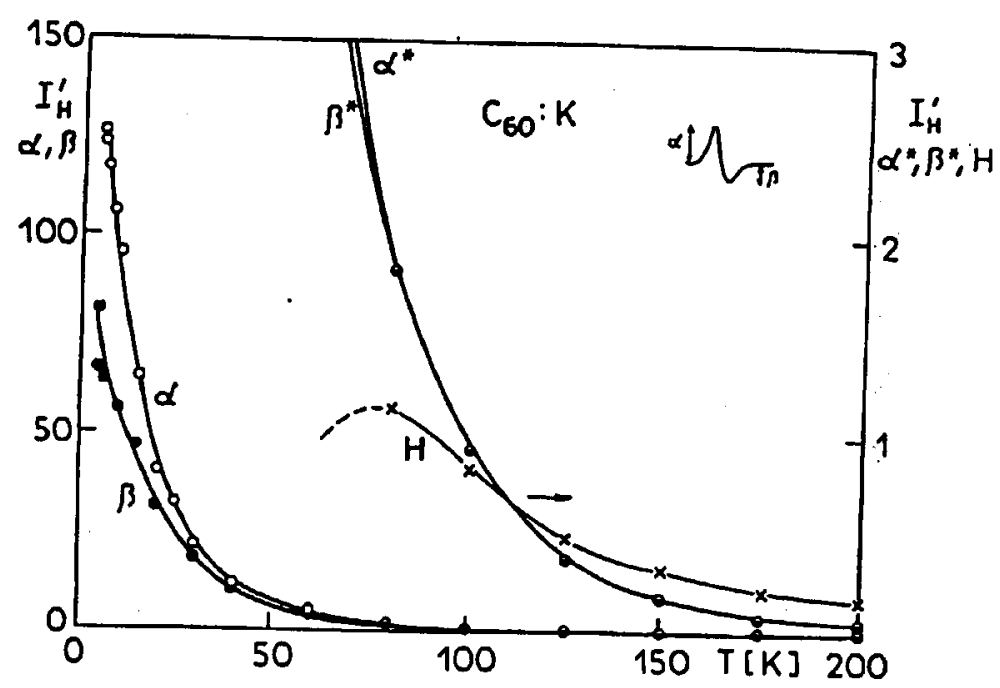

Fig. 4. Temperature dependence of the amplitude of the first derivatives of the absorption intensity of a narrow line $(H)$ and two halves of Dyson type (wide) line $\alpha, \beta$ for $\mathrm{C}_{60}: \mathrm{K}$. The right scale meet $H, \alpha^{*}$ and $\beta^{*}\left(\alpha^{*}\right.$ and $\alpha, \beta^{*}$ and $\beta$ are of course the same lines in a different scale).

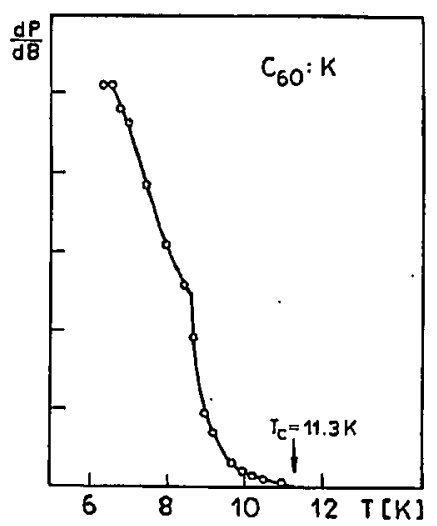

Fig. 5. Temperature dependence of the amplitude of MMMA signal for $\mathrm{C}_{60}: \mathrm{K}$.

\section{Discussion of the results}

On the basis of the results presented by Kukolich et al. [2] and other reports on EPR investigation of fullerenes [1-5] we can reasonably assume the presence of three essentially different paramagnetic centers in fullerenes:

(i) $\left(\dot{\mathrm{C}}_{60}\right)^{+}$ion-radical which is an isolated ionized state of $\mathrm{C}_{60}^{+}$molecule in which the unpaired hole is delocalized on the whole molecule of $\mathrm{C}_{60}$. The spectrum of this ion-radical is characterized by $g$-factor varying from $g_{\mathrm{h}}=2.0023$ to 
$g_{\mathrm{h}} \approx 2.0030$ and a narrow EPR line of the width $2 \Delta B_{1 \mathrm{~s}} \approx 1 \mathrm{Gs}$. The values of $g$-factor and $2 \Delta B_{1 \mathrm{~s}}$ attributed to this center are weakly dependent on temperature.

(ii) $\left(\dot{\mathrm{C}}_{60}\right)^{-}$ion-radical which is an isolated ionized state of $\mathrm{C}_{60}^{-}$molecule in which a trapped electron is delocalized on a whole $\mathrm{C}_{60}$ molecule. Its $g$-factor varies from $g_{\mathrm{t}}=1.9991$ to $g_{\mathrm{t}} \approx 2.0008$ and the EPR line attributed to it is narrow, like for $\left(\dot{\mathrm{C}}_{60}\right)^{+}$, of $\Delta B_{1 \mathrm{~s}}$ taking values between 0.5 and $1.5 \mathrm{Gs}$. The values of $g$-factor and $2 \Delta B_{1 \mathrm{~s}}$ ascribed to this center are weakly dependent on temperature.

(iii) $\left(\dot{\mathrm{C}}_{60}\right)^{y-}$ ion-radical comprised in the complex $\mathrm{Me}^{1+}\left(\dot{\mathrm{C}}_{60}\right)^{y-}$ in which the environment changes distortion of a fullerene molecule affecting both $g$-factor and $2 \Delta B_{1 \mathrm{~s}}$ values. This complex (c) center is characterized by $g_{\mathrm{c}}<g_{0}=2.0023$ which can take values from 2.0005 to 2.0020 and the EPR line attributed to it is wide of $2 \Delta B_{1 \mathrm{~s}}$ of about $10 \mathrm{Gs}$ at room temperature and of about $1 \mathrm{Gs}$ at the temperature of liquid helium. The $g$-factor and $2 \Delta B_{1 \mathrm{~s}}$ linewidth characterizing this center strongly depend on temperature which may be a consequence of the temperature dependence of the $\left(\dot{\mathrm{C}}_{60}\right)^{y-}$ anion radical deformation related to vibrations of the surrounding $\mathrm{Me}^{1+}$ metal cations.

The occurrence of the three so different centers permits explaining the temperature dependence of EPR spectra of fullerenes with very small admixtures of alkali metal ions. For $\mathrm{C}_{60}: \mathrm{Rb}$ the hole centers (line $A$ in Figs. 1 and 2) do not disappear and the temperature dependence of the amplitude of its first derivative of the absorption intensity $I_{A}^{\prime}$ is described by the Curie law. The fact that the width of the line $A$ diminishes with increasing temperature confirms the hole character of the line $A$. Such a dependence is typical of graphite in which hole centers dominate. It is much more difficult to interpret the behavior of centers with trapped electrons. As follows from the EPR spectra analysis the number of these centers shows an activational temperature dependence. Such a temperature dependence of $I_{B}^{\prime}$ can be explained by the following two mechanisms:

1. The interaction between the neighboring centers may lead to the pairing of spins from different $\mathrm{C}_{60}$ ball and the triplet state can occur. The ground state is characterized by the spin $s=0$ while the excited state is paramagnetic. Excited triplet state for $\mathrm{C}_{60}$ and $\mathrm{C}_{70}$ was described previously [5]. In such a case the Curie law includes the temperature dependent number of paramagnetic centers and the amplitude of the EPR line is described by the term $I \approx(1 / T) \exp (-40 / T)$. The presence of this term explains the initial increase in $I_{B}^{\prime}$ with temperature and then the disappearance of the $I_{B}^{\prime}$ line with further temperature increase.

2. The other mechanism of the disappearance of paramagnetic centers in low temperatures could be related to delocalization of the electrons leaving a fullerene $\mathrm{C}_{60}$ molecule and forming electron pairs. Since EPR signal comes only from ion-radicals $\left(\dot{\mathrm{C}}_{60}\right)^{+}$or $\left(\dot{\mathrm{C}}_{60}\right)^{y-}$, delocalization of electrons in the whole crystal can lead to disappearance of the EPR signal. The first mechanism is likely to occur in $\mathrm{C}_{60}: \mathrm{Rb}$ whereas the other can play an essential role in $\mathrm{C}_{60}: \mathrm{K}$ in which below $T=40 \mathrm{~K}$ the metallic conductance has been observed. The latter is strongly related to the superconducting state of fullerites. 


\section{Acknowledgments}

This work was partly supported by the grant 2 P302 00605 of the Committee for Scientific Research.

\section{References}

[1] P. Byszewski, J. Stankowski, Z. Trybuła, W. Kempiński, T. Żuk, J. Mol. Struct. 269, 75 (1992); J. Stankowski, P. Byszewski, W. Kempiński, Z. Trybuła, T. Żuk, Phys. Status Solidi B 178, 221 (1993).

[2] S.G. Kukolich, D.R. Huffman, Chem. Phys. Lett. 182, 263 (1991).

[3] P.J. Krusic, E. Wasserman, B.A. Parkison, B. Malone, E.R. Holler Jr., P.N. Keizer, J.R. Morton, K.F. Preston, J. Am. Chem. Soc. 113, 6274 (1991).

[4] A.A. Zakhidov, A. Ugawa, K. Imaeda, K. Yakushi, H. Inkuchi, Solid State Commun. 79, 939 (1991); Physica C 851, 411 (1991).

[5] M.R. Wasilewski, M.P. O'Neil, K.R. Lykke, M.J. Pellin, D.M. Grune, J. Am. Chem. Soc. 113, 2774 (1991).

[6] K. Tanaka, M. Okada, K. Okahara, T. Yamabe, Chem. Phys. Lett. 113, 6274 (1991). 